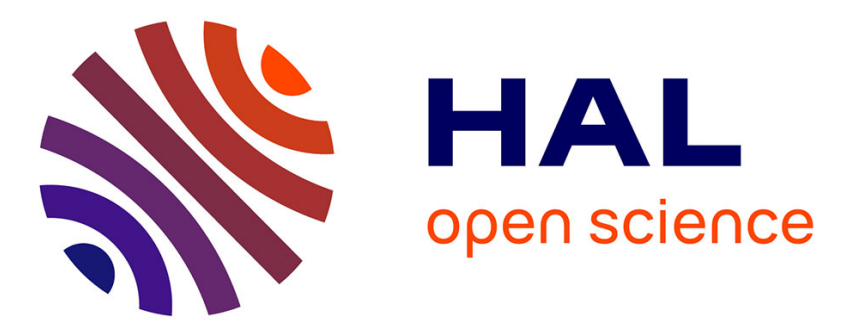

\title{
Using a penalty term to deal with spurious oscillations in second gradient finite elements
}

Marc Soufflet, Gwendal Jouan, Panagiotis Kotronis, Frédéric Collin

\section{To cite this version:}

Marc Soufflet, Gwendal Jouan, Panagiotis Kotronis, Frédéric Collin. Using a penalty term to deal with spurious oscillations in second gradient finite elements. International Journal of Damage Mechanics, 2019, 28 (3), pp.346-366. 10.1177/1056789518769341 . hal-01995276

\section{HAL Id: hal-01995276 https://hal.science/hal-01995276}

Submitted on 13 Feb 2019

HAL is a multi-disciplinary open access archive for the deposit and dissemination of scientific research documents, whether they are published or not. The documents may come from teaching and research institutions in France or abroad, or from public or private research centers.
L'archive ouverte pluridisciplinaire HAL, est destinée au dépôt et à la diffusion de documents scientifiques de niveau recherche, publiés ou non, émanant des établissements d'enseignement et de recherche français ou étrangers, des laboratoires publics ou privés. 


\title{
Using a penalty term to deal with spurious oscillations in second gradient finite elements
}

\author{
Marc Soufflet ${ }^{1,2}$ Gwendal Jouan ${ }^{1,2}$, Panagiotis Kotronis ${ }^{1}$ and Frédéric Collin ${ }^{2}$
}

\begin{abstract}
It is well known that it is necessary to introduce a length scale parameter in a continuum damage mechanics model to correctly simulate strain localization. The second gradient model, a special case of kinematically enriched continua, considers an internal length parameter by taking into account the second order derivatives of the displacements in the virtual power principle. In this paper, we show that the original second gradient finite element of Chambon and co-workers can present spurious oscillations, especially for mode I crack propagation problems. After providing the plane stress second gradient constitutive law, we propose to add a penalty term in the original formulation in order to improve numerical convergence and to avoid spurious oscillations in the local variables distributions. Two numerical examples using classical damage mechanics laws, a three points reinforced concrete beam and a trapezoidal notched specimen are used to test the performance of the formulation. Parametrical studies are also shown on the influence of the penalty parameter. The problem of unrealistic damage spreading for damage values close to one, occurring often in mode I crack propagation problems, is finally discussed.
\end{abstract}

\section{Keywords}

damage mechanics, concrete, second gradient, strain localization, regularization, penalty method

\section{Introduction}

In order to correctly simulate softening in concrete structures, a continuum damage mechanics model with a length scale parameter can be adopted. The scientific literature on continuum mechanics models which include such a length scale is vast and includes different approaches, some of them directly linked to the physical phenomenon leading to softening, while others being more ad hoc, aiming at explicitly introducing the length scale that governs the width of the localization band.

The so called "strongly non-local" models ( see $^{1}$ on the concept of strong and weak non locality) introduce the length scale by explicitly adding a spatial dependency in the constitutive equations, generally via an integral over space. In the case of damage mechanics for example, the damage parameter or the equivalent strain are often defined as an integral of the classical local equivalent variable ${ }^{2}$. Damage can be also introduced in a gradient form, see the works in $^{3},{ }^{4}$ and ${ }^{5}$. The more recent thick level-set model ${ }^{6}$ offers the advantage of a thermodynamically sound approach with a non constant internal length (helping thus to automatically control the evolution of the localization zone) and a smooth transition from damage to fracture. A non constant internal length is also introduced in ${ }^{7}, 8$ and ${ }^{9}$. Finally, the link between a discrete and a nonlocal continuum has been recently studied in ${ }^{10}$.

The kinematically enriched models, whether micromorphic or of the higher order gradient type (see for example ${ }^{11}$ for a classification), add an additional kinematic description of the continuum that implicitly introduces a length scale and thus makes them suitable for strain localization problems. It should be noted that this enriched description can modify the behavior of the structure even before the onset of localization (by introducing for example a size effect). In fact, these generalized continua were not originally specifically developed to deal with strain localization. They can be traced back to the work of the Cosserat brothers in the early $\mathrm{XX}^{\mathrm{ieth}}$ century, ${ }^{12}$. The idea was then to introduce additional

\footnotetext{
${ }^{1}$ Ecole Centrale de Nantes, Université de Nantes, CNRS, GeM (Institut de Recherche en Génie Civil et Mécanique) 1 rue de la Noë, BP 92101, 44321, Nantes, cedex 3, France.

${ }^{2}$ ArGEnCo Department, University of Liège, Allée de la Découverte 9, 4000 Liège, Belgium
}

\section{Corresponding author:}

Panagiotis Kotronis, Ecole Centrale de Nantes, Institut de Recherche en Génie Civil et Mécanique (GeM), 1 rue de la Noë, Nantes, France.

Email: Panagiotis.Kotronis@ec-nantes.fr 
rotational degrees of freedom in the continuum medium. In the sixties, ${ }^{13},{ }^{14}$ and ${ }^{15}$ developed their own versions of kinematically enriched continua. Later on, these approaches were somewhat unified by Germain in a general framework using the virtual power principle, ${ }^{16}$. Applications of these early works dealt, among other things, with wave dispersion, boundary layer effects (surface tension) and piezoelectric materials (see ${ }^{17}$ for a detailed history of the generalized continuum mechanics). One of the earliest use of generalized continua as a way to regularize the problem of strain localization was introduced by ${ }^{18}$ who adopted a Cosserat type continuum, well suited for granular materials, to correctly model shear banding.

Several years latter, Chambon, Caillerie and Hassan gave a closed form solution for the one dimensional problem of strain localization in a bar with a bilinear type constitutive law in a second gradient continuum, ${ }^{19}$. This was soon followed with several applications for plane strain shear banding ${ }^{20},{ }^{21},{ }^{22},{ }^{23}$ mostly in soils, using constitutive models based on plasticity. Similar approaches were also recently used in the framework of damage mechanics combined with homogenization techniques by ${ }^{24},{ }^{25}$, see also ${ }^{26},{ }^{27},{ }^{28}$ and ${ }^{29}$ for application in concrete structures.

Different choices are possible in order to introduce a dissipative mechanism (whether damage, plasticity or a coupling of both) within an enriched kinematics medium. The general framework is now well established for elastoplastic constitutive laws (see for example ${ }^{30}$ and $^{31}$ ) even if the best suited formulation is not always evident. In the case of damage mechanics however, often adopted to simulate the behavior of concrete structures, a similar general framework is to our knowledge still lacking.

The purpose of this paper is first to illustrate the spurious oscillations that can sometimes appear when using the original second gradient finite element of Chambon and co-workers ${ }^{30},{ }^{20}$, especially for mode I crack propagation problems, often occurring in concrete. After providing the plane stress second gradient elastic constitutive law, the original formulation is improved by adding a penalty term in order to enforce the adopted kinematic condition. The ability of the improved formulation to correctly simulate elastic damage induced strain localization under plane stress conditions is studied with two examples: a three points reinforced concrete bending beam test and a trapezoidal notched specimen. Parametrical studies are performed to study the influence of the penalty parameter. Finally, the problem of unrealistic damage spreading occurring mostly in mode I crack propagation problems is highlighted and discussed.
The following notations are adopted hereafter: the Latin indexes ( $i$ to $r$ ) vary from 1 to 3 , the upper script ${ }^{\star}$ represents a virtual variable, the partial derivatives are denoted as $u_{i, j}=\partial u_{i} / \partial x_{j}, u_{i, j k}=\partial^{2} u_{i} / \partial x_{j} \partial x_{k}$ and $K_{i j k}=u_{i, j k} . D q$ is the normal derivative of any quantity $q$ (i.e. $D q=\frac{\partial q}{\partial x_{k}} n_{k}$ ) and $\delta_{i j}$ the Kronecker symbol.

\section{Second gradient model}

Considering a body of domain $\Omega$ and of boundary $\Gamma$, the general formulation of a second gradient model using the virtual power principle can be written as follows ${ }^{19},{ }^{20}$ :

$$
\int_{\Omega}\left(\sigma_{i j} u_{i, j}^{\star}+\Sigma_{i j k} u_{i, j k}^{\star}\right) d \Omega=\int_{\Gamma}\left(p_{i} u_{i}^{\star}+P_{i} D u_{i}^{\star}\right) d \Gamma,
$$

where $\sigma_{i j}$ is the macro stress (the classical second order stress tensor), $\Sigma_{i j k}$ the double stress (a third order tensor) and $p_{i}$ and $P_{i}$ respectively the first (classical) and second order surface traction (first order tensors).

\section{The original finite element formulation}

The displacement field needs to be of class $C^{m-1}$ for the approximate finite element solution, where $m$ is the highest order of derivation appearing in the weak form of the equilibrium $^{32}$. For the second gradient model, this implies the use of finite elements with $C^{1}$ continuity. Such elements have been used in the $1 D$ case by ${ }^{19}$ but are more difficult to construct for $2 D$ and $3 D$ problems (see however ${ }^{33}$ and $^{34}$ ).

One way to circumvent the difficulties of the $C^{1}$ continuity is to adopt a mixed formulation and to interpolate the gradient independently from the displacement field, ${ }^{20},{ }^{35}$. More specifically, the displacement field $u_{i}$ and its gradient noted $v_{i j}$ are both interpolated with $C^{0}$ functions since only first order derivatives appear in the weak formulation provided either by the virtual power principle of a general micromorphic media with a kinematic constrain ${ }^{30}$ or by integration of the strong form of the equilibrium equations ${ }^{35}$. In the former case, we can directly write:

$$
\begin{array}{r}
\int_{\Omega}\left(\sigma_{i j} u_{i, j}^{\star}+\lambda_{i j}\left(v_{i j}^{\star}-u_{i, j}^{\star}\right)+\Sigma_{i j k} v_{i j, k}^{\star}\right) d \Omega= \\
\int_{\Gamma}\left(p_{i} u_{i}^{\star}+P_{i} v_{i j}^{\star} n_{j}\right) d \Gamma
\end{array}
$$

with the added kinematic constraint (in weak form) :

$$
\int_{\Omega} \lambda_{i j}^{\star}\left(v_{i j}-u_{i, j}\right) d \Omega=0
$$

where $\lambda_{i j}$ is the field of Lagrange multipliers associated with the kinematic constraint. For the second gradient model, ${ }^{20}$ 


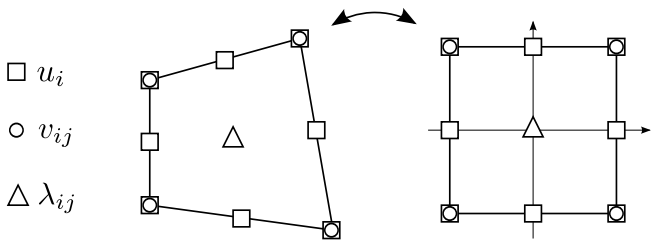

Figure 1. The $2 \mathrm{D}$ second gradient finite element ${ }^{36}$

and ${ }^{36}$ originally developed a 9 noded finite element with the displacement field interpolated by biquadratic functions of the Serendipity type and the gradient field by bilinear functions. Lagrange multipliers are uniform on the element (calculated only on the node in the middle) while numerical integration is performed using a classical Gauss scheme, ${ }^{36}$.

\section{Plane stress linear elastic second gradient constitutive law}

Two constitutive laws are needed in the second gradient model, one for the first gradient part and one for the second gradient part. The constitutive law for $\sigma_{i j}$ depends on the first gradient of the displacements while $\Sigma_{i j k}$ is a function of the second gradient of the displacements, ${ }^{37}$. We present in the following the derivation of the second order linear elastic plane stress constitutive law, starting from the first order classical one.

Consider a bidimensional problem in the Cartesian coordinate system $\left(x_{1}, x_{2}\right)$ and a homogeneous isotropic elastic first gradient constitutive law:

$$
\sigma_{i j}=\lambda \varepsilon_{k k} \delta_{i j}+2 \mu \varepsilon_{i j}
$$

where $\lambda$ and $\mu$ are the Lamé coefficients.

Under the plane stress hypothesis, all components of the stress tensor $\sigma_{i j}$ depending on the out of plane vector $x_{3}$ (i.e. $\sigma_{13}, \sigma_{23}$ and $\sigma_{33}$ and their symmetric parts $\sigma_{32}$ and $\sigma_{31}$ ) are zero. Moreover, the remaining three components $\sigma_{11}, \sigma_{22}$ and $\sigma_{12}$ are functions of $x_{1}$ and $x_{2}$ but are independent of $x_{3}$, i.e. they do not vary through the thickness, ${ }^{38}$. As usual, Eq. (4) combined with the plane stress assumption implies that $\varepsilon_{31}=\varepsilon_{13}=0$ and $\varepsilon_{32}=\varepsilon_{23}=0$, while the expression of the orthogonal stress $\sigma_{33}$ (Eq. (5)) gives $\varepsilon_{33}$ proportional to the bidimensional strains $\varepsilon_{11}$ and $\varepsilon_{22}$ as follows:

$$
\begin{gathered}
0=\sigma_{33}=(\lambda+2 \mu) \varepsilon_{33}+\lambda\left(\varepsilon_{11}+\varepsilon_{22}\right) \quad \Rightarrow \\
\varepsilon_{33}=-\frac{\lambda}{\lambda+2 \mu}\left(\varepsilon_{11}+\varepsilon_{22}\right)
\end{gathered}
$$

Under the small perturbation hypothesis we have $\varepsilon_{i j}=$ $\frac{1}{2}\left(u_{i, j}+u_{j, i}\right)$ and therefore all the strain components depend only on the in plane $\left(x_{1}, x_{2}\right)$ displacement field.
For the linear isotropic elastic case, the relationship between the double stresses and the second gradient of the displacements takes the following form ${ }^{37},{ }^{20}$ :

$$
\begin{aligned}
\Sigma_{r q p}= & a_{1} \frac{1}{2}\left(K_{i p i} \delta_{q r}+K_{i i p} \delta_{q r}+K_{i q i} \delta_{p r}+K_{i i q} \delta_{p r}\right) \\
& +a_{2} \frac{1}{2}\left(K_{p i i} \delta_{q r}+\delta_{p r} K_{q i i}+K_{i r i} \delta_{p q}+K_{i i r} \delta_{p q}\right) \\
& +2 a_{3} \delta_{p q} K_{r i i} \\
& +a_{4}\left(K_{r q p}+K_{r p q}\right) \\
& +a_{5} \frac{1}{2}\left(K_{q r p}+K_{q p r}+K_{p r q}+K_{p q r}\right)
\end{aligned}
$$

where $K_{i j k}=u_{i, j k}$ and $a_{1}$ to $a_{5}$ five independent material parameters.

Under the plane stress hypothesis, all components of the double stress tensor $\Sigma_{i j k}$ depending on the out of plane vector $x_{3}$ are zero. $\Sigma_{i j k}$ has therefore only 8 components $\left(\Sigma_{111}, \Sigma_{112}\right.$, $\left.\Sigma_{121}, \Sigma_{122}, \Sigma_{211}, \Sigma_{212}, \Sigma_{221}, \Sigma_{222}\right)$. As already mentioned, the displacement field is independent of $x_{3}$. Furthermore, the second gradient strains related to the zero first gradient strains $\varepsilon_{i j}$ are also equal to zero. The strain gradients $K_{i j k}$, defined as higher order derivatives of the strains $u_{i, j}$ are therefore calculated as follows:

$\left\{\begin{aligned} \frac{1}{2}\left(K_{131}+K_{311}\right)=\frac{1}{2}\left(u_{1,31}+u_{3,11}\right)=\varepsilon_{13,1}=\varepsilon_{31,1}=0 \\ \frac{1}{2}\left(K_{132}+K_{312}\right)=\frac{1}{2}\left(u_{1,32}+u_{3,12}\right)=\varepsilon_{13,2}=\varepsilon_{31,2}=0 \\ \frac{1}{2}\left(K_{133}+K_{313}\right)=\frac{1}{2}\left(u_{1,33}+u_{3,13}\right)=\varepsilon_{13,3}=\varepsilon_{31,3}=0 \\ \frac{1}{2}\left(K_{231}+K_{321}\right)=\frac{1}{2}\left(u_{2,31}+u_{3,21}\right)=\varepsilon_{23,1}=\varepsilon_{32,1}=0 \\ \frac{1}{2}\left(K_{232}+K_{322}\right)=\frac{1}{2}\left(u_{2,32}+u_{3,22}\right)=\varepsilon_{23,2}=\varepsilon_{32,2}=0 \\ \frac{1}{2}\left(K_{233}+K_{323}\right)=\frac{1}{2}\left(u_{2,33}+u_{3,23}\right)=\varepsilon_{23,3}=\varepsilon_{32,3}=0\end{aligned}\right.$

We can thus identify that the following 12 tensorial components $\left(K_{311}, K_{312}, K_{313}, K_{131}, K_{132}, K_{133}, K_{321}, K_{322}\right.$, $K_{323}, K_{231}, K_{232}$ and $\left.K_{233}\right)$ and their symmetric parts $\left(K_{131}=\right.$ $K_{113}, K_{313}=K_{331}, K_{323}=K_{332}, K_{132}=K_{123}, K_{231}=K_{213}$ and $\left.K_{232}=K_{223}\right)$ are equal to zero. Looking at the out of plane strain we can also deduce that $K_{333}=0$. In total, 19 out of the 27 components of the constitutive tensor of the second gradient constitutive law are equal to zero and one finally finds the same equation as under the plane strain hypothesis, ${ }^{30}$. The number of parameters of a constitutive model is a crucial issue. While few parameters are sometimes not able to reproduce precisely the material behavior, they can allow a clearer view of what is happening and ease the calibration procedure. For these reasons, ${ }^{20}$ chose to work with only one parameter $B$. The plane stress second gradient 
constitutive law, linking the double stresses $\Sigma_{i j k}$ to the second order strain $K_{i j k}$ is thus written as:

$$
\left[\begin{array}{l}
\Sigma_{111} \\
\Sigma_{112} \\
\Sigma_{121} \\
\Sigma_{122} \\
\Sigma_{211} \\
\Sigma_{212} \\
\Sigma_{221} \\
\Sigma_{222}
\end{array}\right]=\left[\begin{array}{cccccccc}
B & 0 & 0 & 0 & 0 & \frac{B}{2} & \frac{B}{2} & 0 \\
0 & \frac{B}{2} & \frac{B}{2} & 0 & -\frac{B}{2} & 0 & 0 & \frac{B}{2} \\
0 & \frac{B}{2} & \frac{B}{2} & 0 & -\frac{B}{2} & 0 & 0 & \frac{B}{2} \\
0 & 0 & 0 & B & 0 & -\frac{B}{2} & -\frac{B}{2} & 0 \\
0 & -\frac{B}{2} & -\frac{B}{2} & 0 & B & 0 & 0 & 0 \\
\frac{B}{2} & 0 & 0 & -\frac{B}{2} & 0 & \frac{B}{2} & \frac{B}{2} & 0 \\
\frac{B}{2} & 0 & 0 & -\frac{B}{2} & 0 & \frac{B}{2} & \frac{B}{2} & 0 \\
0 & \frac{B}{2} & \frac{B}{2} & 0 & 0 & 0 & 0 & B
\end{array}\right]\left[\begin{array}{l}
K_{111} \\
K_{112} \\
K_{121} \\
K_{122} \\
K_{211} \\
K_{212} \\
K_{221} \\
K_{222} \\
(8)
\end{array}\right]
$$

Other assumptions are of course possible. ${ }^{39}$ proposed for example a "dilation second gradient" model, imposing a different relation between the 5 independent parameters. It should be noted however that the choices made to reduce the number of parameters can influence the ability of the model to reproduce the different localization modes. For example, a "second gradient Cosserat" model can be derived from the general second gradient model ${ }^{30}$ but this model is unable to deal with mode I localization. The calibration of the material parameter $B$ is often difficult. Analytical solutions, experimental results or $1 \mathrm{D}$ calculations can be adopted, see a proposed methodology in ${ }^{29}$.

\section{First gradient constitutive law}

In their work, Chambon and co-workers often consider the first gradient constitutive law decoupled from the second gradient one. With this assumption, it is straightforward to adopt classical constitutive laws (e.g. based on plasticity or damage mechanics) for the first gradient part. The authors showed that this framework regularizes stain localization problems as the second gradient linear elastic law acts as a penalty term for the strain gradients, ensuring that strains localize in a band of finite size. They provided several applications in soil mechanics ${ }^{20},{ }^{22},{ }^{40},{ }^{41}$.

In this article, the first and second gradient constitutive laws are considered decoupled, a classical damage mechanics law is used for the first gradient part while the second gradient law is linear elastic. Damage acts therefore only on the first gradient constitutive law and is a function of the first gradient of the displacements. It should be noted however that the introduction of damage on the second gradient part has been studied by different authors, ${ }^{42},{ }^{24},{ }^{25} 27 . \mathrm{In}^{42}$ and for a 1D case,
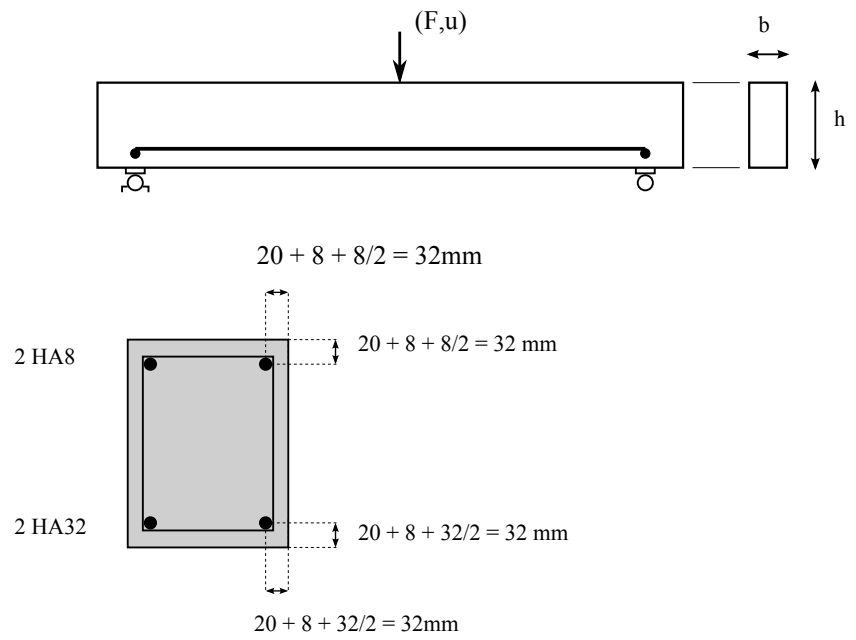

Figure 2. Three points bending test: beam dimensions and steel reinforcement ${ }^{43}, 44$

it is shown that a carefully formulated coupling of the first and the second gradient laws leads to an a priori control of the width evolution (constant, increase or decrease) of the localisation zone.

\section{Improved formulation of second gradient model}

\section{Spurious oscillations observed with the original formulation}

In order to illustrate the spurious oscillations problem that can occur when using the original second gradient formulation, a three points bending test on a reinforced concrete beam is simulated hereafter. The concrete beam has the following geometrical characteristics: thickness $b=200 \mathrm{~mm}$, height $h=500 \mathrm{~mm}$ and span $5000 \mathrm{~mm}$. The tested beam and the steel reinforcement are shown in figure 2.

The finite element mesh consists of 5180 elements, 4148 of which are second gradient elements and 1032 truss elements representing the horizontal reinforcement. The average size of the concrete elements is of $0.02 \mathrm{~m} \times 0.035 \mathrm{~m}$. The two supports (the two points at the bottom of the specimen, see figure 2) are blocked vertically while the right support is also blocked horizontally. For the finite element calculations, monotonically increased displacements are applied at the upper part of the beam through an elastic plate, which is very stiff compared to the other materials.

At both supports at the bottom of the beam and on the upper part, where the displacements are applied, an elastic linear law is introduced to prevent any artificial numerical damage. A classical damage mechanics law is used for the first gradient constitutive law ${ }^{45}$, where the scalar damage parameter $D$ is 


\begin{tabular}{ccccccc}
\hline $\mathrm{E}(\mathrm{GPa})$ & $\epsilon_{D 0}$ & $A_{t}$ & $B_{t}$ & $A_{c}$ & $B_{c}$ & $\beta$ \\
\hline 37.2 & $9.1 \mathrm{E}-05$ & 0.7 & 6800 & 0.42 & 780 & 1.1
\end{tabular}

Table 1. 3 points bending test: concrete material parameters ${ }^{29}$

a function of an internal variable $\kappa$ depending on the loading history and on the equivalent strain $\epsilon_{e q}$, through the definition of a loading function (Eq. (9)) and the Kuhn-Tuker conditions (Eq. (10)):

$$
\begin{gathered}
f\left(\epsilon_{e q}, \kappa\right)=\epsilon_{e q}-\kappa \\
f \leq 0 ; \quad \dot{\kappa} \geq 0 ; \quad f \dot{\kappa}=0
\end{gathered}
$$

The equivalent strain ${ }^{45}$ is defined by:

$$
\epsilon_{e q}=\sqrt{\sum_{i=1}^{3}\left\langle\epsilon_{i}\right\rangle_{+}^{2}}
$$

where $\left\langle\epsilon_{i}\right\rangle_{+}$is the positive part of the principal strains.

The damage evolution takes into account the contribution of traction and compression to better represent the concrete behavior $^{45}$ :

$$
D=\gamma^{\beta} d_{t}(\kappa)+(1-\gamma)^{\beta} d_{c}(\kappa)
$$

where $\gamma$ is a function of the equivalent strain $\epsilon_{e q}$, the positive part of the principal strains $\left\langle\epsilon_{i}\right\rangle_{+}$and of the principal strains due to positive principal stresses $\epsilon_{i}^{t}$ :

$$
\gamma=\sum \frac{\epsilon_{i}^{t}<\epsilon_{i}>_{+}}{\epsilon_{e q}}
$$

The evolutions of $d_{t}$ and $d_{c}$ are given by :

$$
\begin{aligned}
& d_{t}=1-\frac{\kappa_{i}\left(1-A_{t}\right)}{\kappa}-\frac{A_{t}}{e^{\left(B_{t}\left(\kappa-\kappa_{i}\right)\right)}} \\
& d_{c}=1-\frac{\kappa_{i}\left(1-A_{c}\right)}{\kappa}-\frac{A_{c}}{e^{\left(B_{c}\left(\kappa-\kappa_{i}\right)\right)}}
\end{aligned}
$$

where $A_{t}, A_{c}, B_{t}, B_{c}$ and $\beta$ are material constants. The concrete material parameters of the first gradient law are finally provided in table 1 , see also ${ }^{29}$.

An isotropic linear elastic constitutive law is adopted for the second gradient part depending on a single material parameter $B=1.5 M N$, following Eq. (8). No coupling is assumed between the first and the second gradient constitutive laws.

An elastic perfectly plastic law is used for the reinforcement and a perfect bond is assumed. The steel material parameters

\begin{tabular}{ccc}
\hline $\mathrm{E}(\mathrm{GPa})$ & $\sigma_{y}(\mathrm{MPa})$ & Section $\left(m^{2}\right)$ \\
\hline 195 & 466 & $16.08510^{-4}(2 \mathrm{HA} 32)$
\end{tabular}

Table 2. 3 points bending test: steel parameters (lower part) ${ }^{29}$

\begin{tabular}{ccc}
\hline $\mathrm{E}(\mathrm{GPa})$ & $\sigma_{y}(\mathrm{MPa})$ & Section $\left(m^{2}\right)$ \\
\hline 195 & 466 & $1.005310^{-4}(2 \mathrm{HA} 8)$ \\
\hline
\end{tabular}

Table 3. 3 points bending test: steel parameters (upper part) ${ }^{29}$
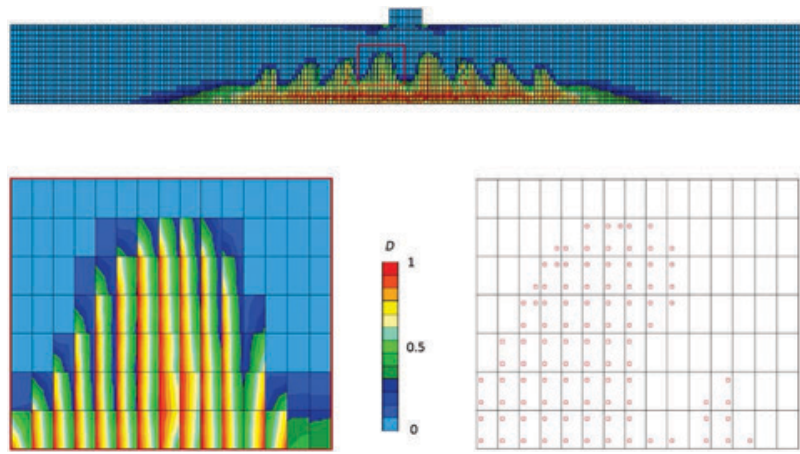

Figure 3. Damage distribution in a three points beam test (top figure). Zoom on damage oscillation problems (left figure) and loading integration points (red rectangles correspond to integration points with an increasing damage variable, right figure)

are given in tables 2 and 3 (where $\sigma_{y}$ the yield stress and HA states for "high adherence"):

$\mathrm{In}^{29}$, we were able to obtain good results for the global force displacement curve. However, a closer look at the damage distribution reveals an oscillation of its value inside the second gradient finite elements. Figure 3 shows the damage distribution in the entire beam as well as two close ups. It can be observed that in the localization bands and inside certain finite elements some integration points present an increasing damage variable while others are unloading.

Looking at the distribution of one component of the derivative of the displacement, say $u_{1,1}$, calculated by deriving the displacement field (figure 4) and comparing it to its corresponding gradient field, $v_{11}$ in this case (figure 5), it is clear that both fields are not equal at each integration point but only on average on the element. Within the localization bands, the values of $u_{i, j}$ may oscillate.

The damage variable, function of the strains computed by deriving the displacement field (and not through the $v_{i j}$ component) can thus oscillate just as the displacement derivatives do. This is due to the fact that the original mixed formulation implies that the constraints imposed by the Lagrange multipliers are verified on average on each element (one Lagrange multiplier per element is adopted). However, on a specific integration point the value of the independently interpolated gradient field $v_{i j}$ can significantly differ from 


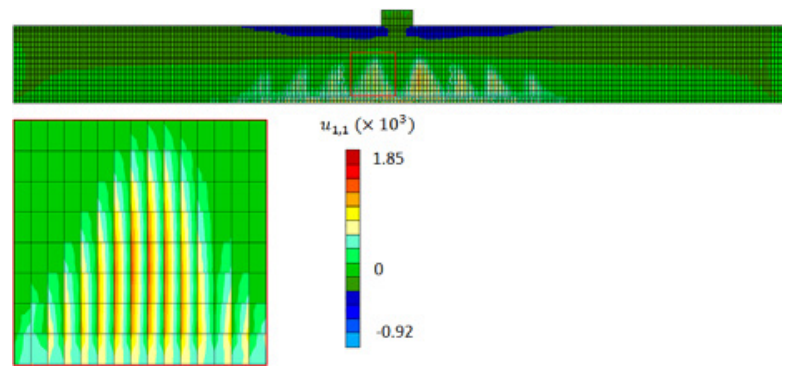

Figure 4. Distribution of the $u_{1,1}$ component (overall and zoomed view) for an imposed displacement of $5 \mathrm{~mm}$

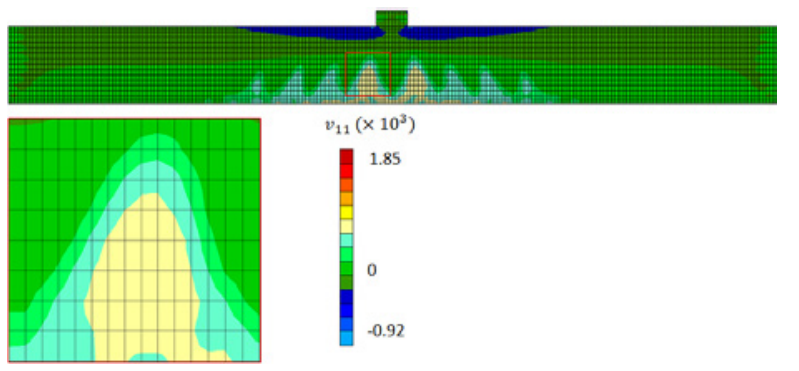

Figure 5. Distribution of the $v_{11}$ component (overall and zoomed view) for an imposed displacement of $5 \mathrm{~mm}$

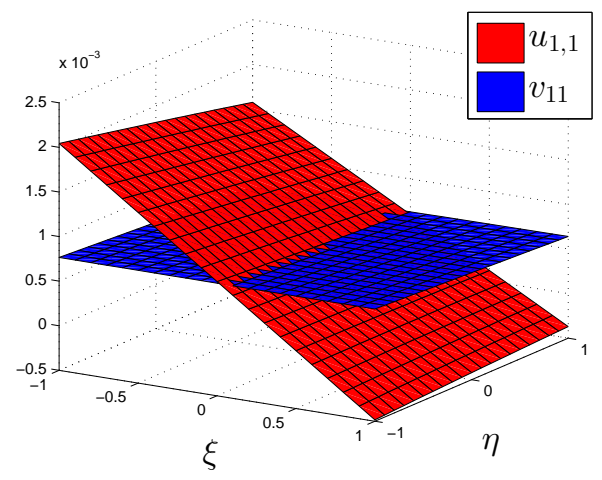

Figure 6. Distribution of $u_{1,1}$ and $v_{11}$ on the original mixed second gradient finite element formulation

the value calculated from the derivative of the displacement field $u_{i}$. The oscillations are therefore a consequence of the chosen finite element formulation. Figure 6 illustrates this situation on a single finite element (where $\xi$ and $\eta$ are the local element coordinates). The distribution of the $v_{11}$ component is different from the distribution of $u_{1,1}$ but the equality of both fields is verified on average (a 2 by 2 Gauss numerical integration scheme is adopted). This can lead to damage oscillation, one of the main sources of the convergence problems encountered in ${ }^{29}$.

\section{Addition of a penalty term}

A way to improve the numerical performance of the original mixed second gradient finite element formulation is to add a penalty term to enforce the kinematic constraint (while keeping the Lagrange multipliers $)^{28}$. A similar approach was proposed by ${ }^{39}$ in the context of plasticity. Noting $C$ the penalty factor, the weak formulation of the problem becomes:

$$
\begin{aligned}
& \int_{\Omega}\left(\sigma_{i j} u_{i, j}^{\star}+\lambda_{i j}\left(v_{i j}^{\star}-u_{i, j}^{\star}\right)+C\left(v_{i j}^{\star}-u_{i, j}^{\star}\right)\left(v_{i j}-u_{i, j}\right)+\Sigma_{i j k} u_{i, j k}^{\star}\right) d \Omega= \\
& \int_{\Gamma}\left(p_{i} u_{i}^{\star}+P_{i} v_{i j, k}^{\star} n_{k}\right) d \Gamma
\end{aligned}
$$

Remark: An alternative technique is to use the continuous field $v_{i j}$, instead of the derivative of the displacement $u_{i, j}$, to compute the equivalent strain used to calculate the damage value of the first gradient constitutive law, (for detailed informations $\mathrm{se}^{28}$ ). In this way, spurious oscillations are avoided. This latter approach, although simpler to implement and providing good results, has the disadvantage of modifying the damage mechanics constitutive law. This approach is not further investigated in this article.

In the next section, two numerical examples are provided to illustrate the performance of the penalty term method: the three points bending test on a reinforced concrete beam revisited and a trapezoidal notched beam.

\section{Numerical applications}

The second gradient model has been mainly used in soil mechanics, where strains localize in shear bands (mode II crack propagation, see for example ${ }^{20},{ }^{22},{ }^{23}$ ). In concrete structures however, cracks often propagate according to mode I and therefore the strain localization phenomenon significantly differs. Two cases of mode I problems are studied hereafter using the second gradient finite element with the penalty term: the reinforced concrete beam submitted to a three points bending test of section and a trapezoidal notched specimen.

In order to study the influence of the penalty factor on the global numerical results (force - displacement curve), a relative error is computed for the two cases, based on the derivative of the displacement field $u_{i, j}$ and the gradient of the displacement field $v_{i j}{ }^{39}$ :

$$
R=\frac{\int_{\Omega}\left(u_{i, j}-v_{i j}\right)^{2} d \Omega}{\int_{\Omega} v_{i j}^{2} d \Omega}
$$

The penalty factor $C$ is adimensionalized by dividing it by the initial Young modulus $E_{0}$ to better compare the results. 


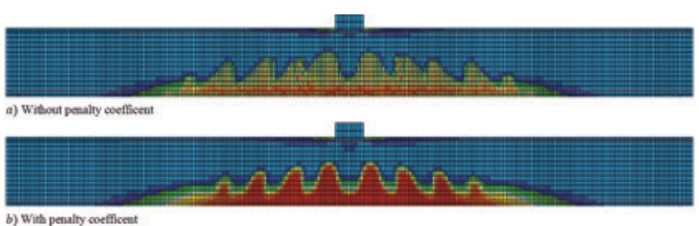

Figure 7. Three points bending test: damage distribution for an imposed displacement of $6 \mathrm{~mm}$ a) without and b) with a penalty factor $\left(C / E_{0}=2.69\right)$

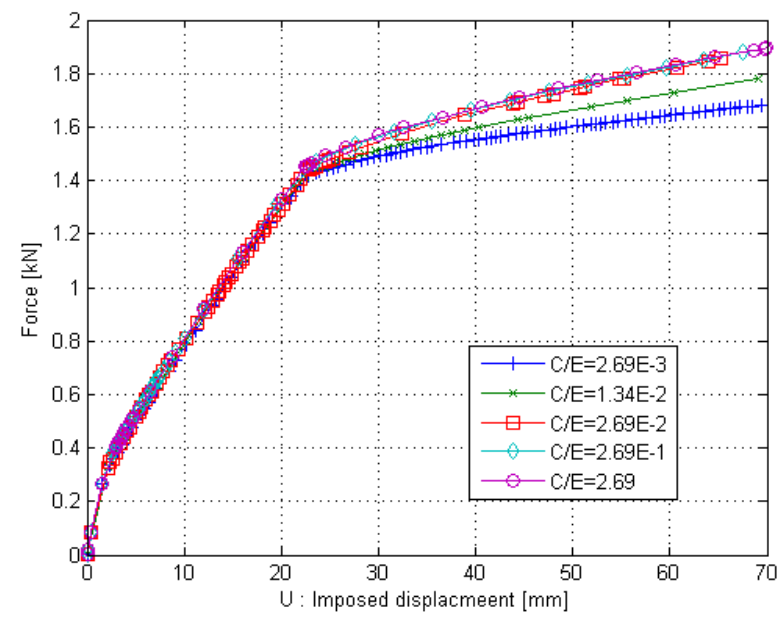

Figure 8. Three points bending test: reaction force vs. imposed displacement for different normalized penalty factors

\section{The three points bending test on a reinforced concrete beam (revisited)}

The three points bending test of section under the plane stress assumption is modelled hereafter using the second gradient finite element with the added penalty term.

Influence of the penalty factor Figure 7 gives the damage distribution corresponding to an imposed displacement $U=$ $6 \mathrm{~mm}$ without and with a penalty factor $\left(C / E_{0}=2.69\right)$. It is clear that the introduction of the penalty factor improves the numerical performance of the model and results to a more smooth and continuous damage distribution.

Figure 8 shows the global force displacement curves for different values of $C / E_{0},\left(2.69 \times 10^{-3}, 1.34 \times 10^{-2}, 2.69 \times\right.$ $10^{-2}, 2.69 \times 10^{-1}, 2.69, E_{0}$ being constant). The global force displacement curves are clearly influenced by the penalty term. However for values of $C / E_{0}$ equal or greater than $2.69 \times 10^{-2}$ the results become nearly identical.

Figure 9 (a) and (b) present the respective relative error (see Eq. (17)) for different penalty factors using classical and logarithmic scales. While figure 9 (a) show a sharp decrease of the relative error with increasing penalty factors, figure 9 (b) illustrates that small penalty factors have little or no effects. Furthermore, an upper threshold for the penalty factors exists

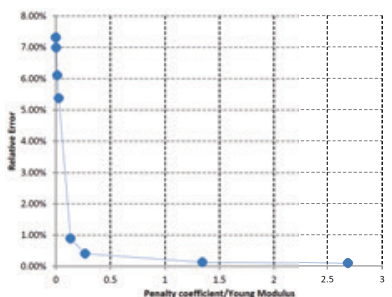

a)

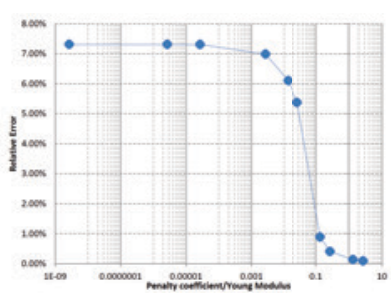

b)
Figure 9. Three points bending test: relative error at $U=6 \mathrm{~mm}$ for different normalized penalty factors in classical a) and in logarithmic scale b)

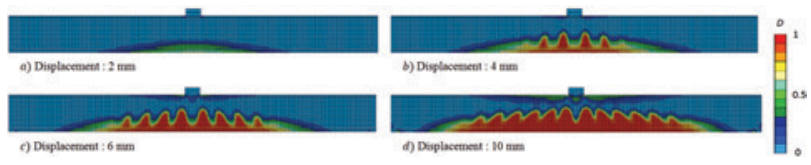

Figure 10. Three points bending test: evolution of the damage distribution for various imposed displacements $\left(C / E_{0}=2.69\right)$

such as beyond it the relative error stays small and almost unchanged. One has to keep in mind however that very high penalty factors can lead to convergence problems.

Evolution of the damage distribution Figure 10 shows the damage distribution for various imposed displacements with a normalized penalty factor $\left(C / E_{0}=2.69\right)$. Damage spreads with increasing loading and new cracks appear (figure 10 $a, b, c)$. They widen until finally they merge so that no individual crack pattern is observed at the bottom of the beam (figure $10 \mathrm{~d}$ ). This is linked to the unrealistic damage spreading occurring for damage values close to 1 (e.g. mode I crack propagation problems). It will be more thoroughly discussed in the section dealing with the evolution of damage distribution for the trapezoidal notched beam.

To further analyse the problem of damage distribution in mode I crack propagation using the penalty term, a trapezoidal notched beam test is presented hereafter.

\section{Trapezoidal notched beam}

Geometry and constitutive laws Consider the notched trapezoidal beam presented in figure 11 with a vertical imposed displacement $U$ applied at the notch lips. This mode I crack propagation test was proposed by ${ }^{46}$.

An isotropic damage mechanics model is again used for the constitutive law of the first gradient part. The internal variable and the equivalent strain are still defined by Eqs. (9), (10) and (11) while the adopted damage evolution has the following form:

$$
D=1-\frac{\kappa_{i}}{\kappa}\left(1-\alpha+\alpha e^{-\beta\left(\kappa-\kappa_{i}\right)}\right)
$$




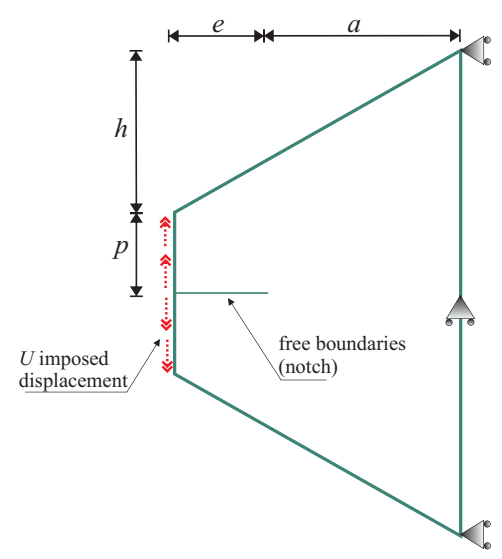

Figure 11. Notched trapezoidal beam: mode I crack propagation test $^{46}$

\begin{tabular}{llccl}
\hline Type & Parameter & & Value & Unit \\
\hline Geometry & height & $h$ & 20 & $\mathrm{~cm}$ \\
& width & $a$ & 10 & $\mathrm{~cm}$ \\
& notch & $e$ & 9 & $\mathrm{~cm}$ \\
& tip & $p$ & 5 & $\mathrm{~cm}$ \\
Elastic part & Young modulus & $E$ & 30 & $\mathrm{GPa}$ \\
& Poisson's coefficient & $v$ & 0.22 & - \\
Damage law & Initial strain & $\kappa_{i}$ & 0.03 & $\%$ \\
& Curve shape & $\alpha$ & 0.99 & \\
& Curve shape & $\beta$ & 2000 & \\
& & & & \\
Second gradient law & Elastic modulus & $B$ & 3.47 & $M N$
\end{tabular}

Table 4. Notched trapezoidal beam: material and geometric parameters

where $\alpha$ and $\beta$ are material parameters controlling the shape of the softening curve and $\kappa_{i}$ the initial value of the internal variable $\kappa$. Eq. (18) results to an exponentially decreasing stress strain curve for the $1 \mathrm{D}$ case. An isotropic linear elastic constitutive law is adopted for the second gradient part depending on a single material parameter $B$, following Eq. (8). No coupling is assumed between the first and the second gradient constitutive laws. All material and geometric parameters are provided in table 4.

Influence of the penalty factor Several ratios $C / E_{0}$ are again considered $\left(3.33 \times 10^{-3}, 3.33 \times 10^{-2}, 3.33 \times 10^{-1}, 1.67\right.$ and $3.33, E_{0}$ being the initial Young modulus) and results are presented in figure 12, in terms of reaction force vs. imposed displacement $U$. For each calculation, three distinct phases are apparent: an elastic linear state, a non-linear phase with a decreasing slope up to the peak value and finally a post peak softening phase. Without the penalty factor, the maximum force is found smaller and results present a more brittle behaviour. Furthermore, several jumps exist during the softening phase, corresponding to abrupt relaxations. Using high normalized penalty factors $\left(3.33 \times 10^{-2}, 3.33 \times 10^{-1}\right.$, 1.67 and 3.33 ), the maximum force is increased by $25 \%$

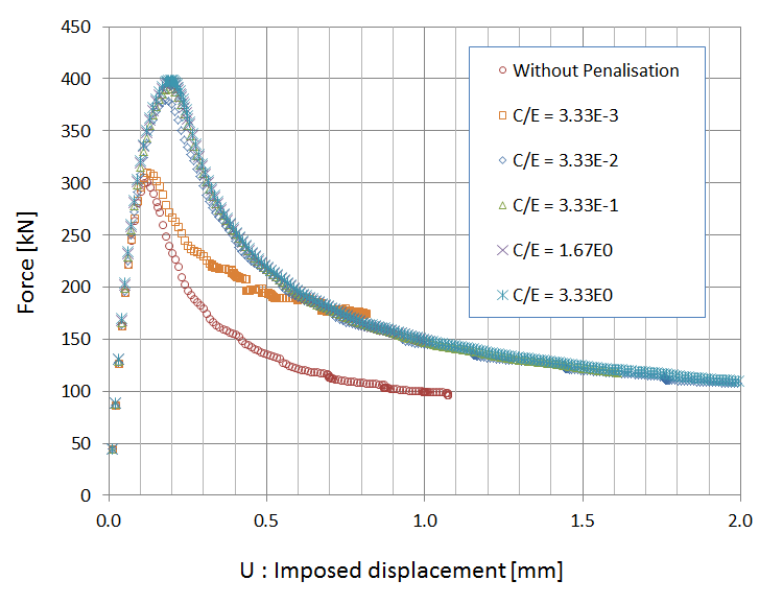

Figure 12. Notched trapezoidal beam: reaction force vs. imposed displacement for different normalized penalty factors

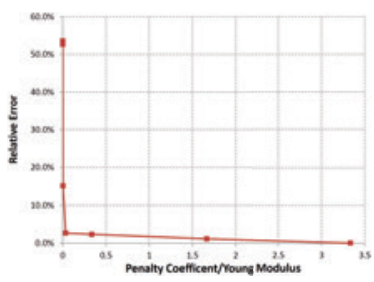

(a)

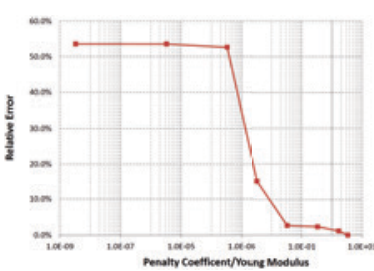

(b)
Figure 13. Notched trapezoidal beam: relative error at $U=0.25 \mathrm{~mm}$ for different penalty factors in classical (a) and in logarithmic scale (b)

and the softening phase becomes smoother. Results become virtually identical for normalized penalty factors greater than $3.33 \times 10^{-1}$. For small values of normalized penalty factors (i.e. equal or lower to $3.33 \times 10^{-3}$ ) the correct damage distribution and evolution are not recovered.

Figure 13 (a) presents the relative error for different normalized penalty factors just before the peak load ( $U$ : $0.25 \mathrm{~mm}$ ). Figure 13 (b) presents the same error evolution curve using a logarithmic scale. The first point of both graphs corresponds to computations without a penalty factor $(C=0)$ and presents a relative error of approximately $51 \%$. As the penalty factor increases, the error sharply decreases (up to a normalized value of $C / E_{0}=3.33 \times 10^{-2}$ ) and then decreases slowly $\left(C / E_{0}\right.$ between $3.33 \times 10^{-1}$ and 3.33$)$. The numerical computation does not converge for $C / E_{0}$ equal to or greater than 3.33. It is therefore obvious that a carefully calibrated penalty factor significantly improves the quality of the numerical results.

Evolution of the damage distribution A realistic reproduction of the evolution of strain localization zone is crucial. Non local integral type regularization and the closely related implicit gradient type present several limitations ${ }^{5},{ }^{47},{ }^{48},{ }^{49}, 50$. 


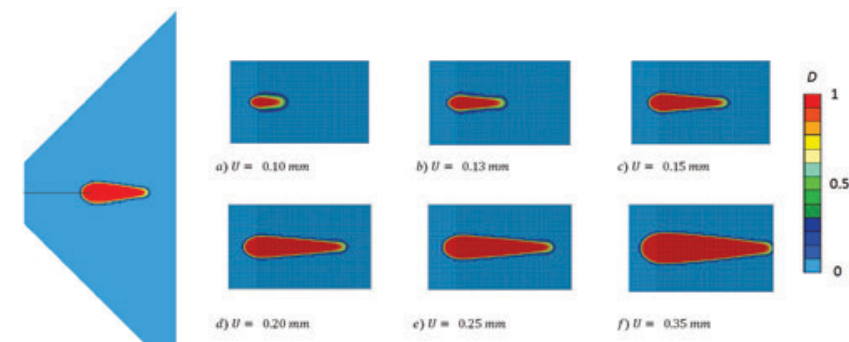

Figure 14. Notched trapezoidal beam: (left) damage distribution for $U=0.20 \mathrm{~mm}$ and (right) close up at the notch tip for various imposed displacements $\left(C / E_{0}=3.33\right)$
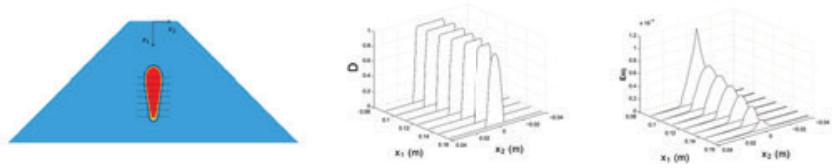

Figure 15. Notched trapezoidal beam: damage and equivalent strains around the crack for $U=0.20 \mathrm{~mm}$ and $C / E_{0}=3.33$

Similar problems were identified for the second gradient model $^{51}, 26,42,22,29$.

Figure 14 shows the damage distribution in the trapezoidal notched beam for an imposed displacement $U=0.20 \mathrm{~mm}$ and for $C / E_{0}=3.33 \times 10^{-2}$ and a close up at the notch tip for various imposed displacements. Spreading of damage behind the crack front is clearly visible. This is similar to the widening of the damage zone for the implicit gradient model studied by de Borst et al. ${ }^{5}$.

In our case, the origin of spreading is better understood when looking at the damage propagation as a series of $1 D$ problems in the direction perpendicular to the crack propagation: figure 15 shows the equivalent strain $\epsilon_{e q}$ and the damage $D$ distributions along the axis orthogonal to the crack. The width of the localization zone increases as we move away from the damage front. As mentioned in ${ }^{29}$ and $^{22}$, for an (essentially) $1 D$ problem the width of the localization zone is approximately equal to $l_{s}=2 \pi \sqrt{\frac{B}{\left|G_{t g}\right|}}$, where $G_{t g}$ is the tangent modulus of the softening part of the first gradient law. The chosen damage law implies that the tangent modulus decreases exponentially (in absolute value) with increasing damage $D$ and thus the localization zone increases without bounds behind the damage front. This feature of the second gradient model is more critical in the context of a mode I propagation than in a mode II (the principal mode of localization in soils), because in the former case the damage gradient is high at the crack front and high levels of damage are reached as soon as the crack propagates. For the mode II case, plasticity or damage stay more or less constant within the band (see for example ${ }^{20},{ }^{22},{ }^{23}$ ).
For the three points bending test presented previously, the damage spreading problem is also apparent (see figure 10). This behaviour becomes problematic as cracks merge and cannot be individually discerned. It should be however highlighted that this happens for very severe loadings.

\section{Conclusions}

The second gradient model introduced by Chambon and co-workers $\left({ }^{30},{ }^{20}\right)$ is used as a regularization method in the context of damage induced strain localization. After the presentation of the plane stress second gradient constitutive law, the numerical performance of the orignal formulation of the second gradient finite element is improved by introducing an additional penalty term in the Lagrangian mixed formulation. A parametrical study is performed on the influence of the penalty factor and the evolution of the damaged distribution in a mode I crack propagation is investigated. Examples are provided considering a reinforced concrete beam and a trapezoidal notched beam. The numerical applications show the ability of the improved second gradient formulation to reproduce damage evolution. The problem of unrealistic damage spreading for damage values close to one, occurring often in mode I crack propagation problems, is finally discussed.

\section{References}

1. Maugin G. Nonlocal theories or gradient-type theories-a matter of convenience. Archiv of Mechanics, Archiwum Mechaniki Stosowanej 1979; 31: 15-26.

2. Pijaudier-Cabot G and Bazant ZP. Nonlocal damage theory. Journal of Engineering Mechanics 1987; 113(10): 1512-1533.

3. Peerlings R, De Borst R and De Vree J. Gradient enhanced damage for quasi-brittle materials. International Journal for Numerical Methods in Engineering 1996; 39: 3391-3403.

4. Peerlings R, Geers $M$, De Borst $R$ et al. A critical comparison of nonlocal and gradient-enhanced softening continua. International Journal of solids and Structures 2001; 38(44-45): 7723-7746.

5. de Borst R, Geers M, Peerlings R et al. Some remarks on gradient and nonlocal damage theories. In Voyiadjis G, Woody Ju JW and Chaboche J (eds.) Damage Mechanics in Engineering Materials, Studies in Applied Mechanics, volume 46. Elsevier, 1998. pp. 223 - 236. DOI:10.1016/ S0922-5382(98)80044-5.

6. Moës N, Stolz C, Bernard PE et al. A level set based model for damage growth: the thick level set approach. International Journal for Numerical Methods in Engineering 2011; 86(3): 358-380. 
7. Pijaudier-Cabot G, Haidar K and Dubé JF. Non-local damage model with evolving internal length. International journal for numerical and analytical methods in geomechanics 2004; 28(78): 633-652.

8. Giry C, Dufour F and Mazars J. Stress-based nonlocal damage model. International Journal of Solids and Structures 2011; 48(25-26): 3431-3443.

9. Rastiello G, Giry C, Gatuingt F et al. From diffuse damage to strain localization from an eikonal non-local (enl) continuum damage model with evolving internal length. Computer Methods in Applied Mechanics and Engineering 2018; 331: 650-674.

10. Challamel N, Picandet V and Pijaudier-Cabot G. From discrete to nonlocal continuum damage mechanics: Analysis of a lattice system in bending using a continualized approach. International Journal of Damage Mechanics 2015; 24(7): 983-1012.

11. Forest $\mathrm{S}$ and Sievert R. Nonlinear microstrain theories. International Journal of Solids and Structures 2006; 43(24): 7224 - 7245. DOI:10.1016/j.jjsolstr.2006.05.012. Sizedependent Mechanics of Materials.

12. Cosserat E, Cosserat F, Brocato $\mathrm{M}$ et al. Théorie des corps déformables. A. Hermann Paris, 1909.

13. Mindlin R. Micro-structure in linear elasticity. Archive for Rational Mechanics and Analysis 1964; 16(1): 51-78.

14. Toupin R. Elastic materials with couple-stresses. Archive for Rational Mechanics and Analysis 1962; 11(1): 385-414.

15. Eringen A. Linear theory of micropolar elasticity. Technical report, Purdue University Lafayette in school of aeronautics and astronautics, 1965

16. Germain P. The method of virtual power in continuum mechanics. part 2: Microstructure. SIAM Journal on Applied Mathematics 1973; 25(3): 556-575.

17. Maugin GA. Generalized continuum mechanics: What do we mean by that? In Mechanics of Generalized Continua. Springer, 2010. pp. 3-13.

18. Muhlhaus $\mathrm{H}$ and Vardoulakis I. The thickness of shear bands in granular materials. Geotechnique 1987; 37(3): 271-283.

19. Chambon R, Caillerie D and Hassan NE. One-dimensional localisation studied with a second grade model. European Journal of Mechanics - A/Solids 1998; 17(4): 637 - 656. DOI: 10.1016/S0997-7538(99)80026-6.

20. Matsushima T, Chambon R and Caillerie D. Large strain finite element analysis of a local second gradient model: application to localization. International journal for numerical methods in engineering 2002; 54(4): 499-521.

21. Collin F, Chambon R and Charlier R. A finite element method for poro mechanical modelling of geotechnical problems using local second gradient models. International Journal for Numerical Methods in Engineering 2006; 65(11): 1749-1772.
22. Kotronis P, Al Holo S, Bésuelle P et al. Shear softening and localization: Modelling the evolution of the width of the shear zone. Acta Geotechnica 2008; 3(2): 85-97.

23. Sieffert $\mathrm{Y}, \mathrm{Al}$ Holo $\mathrm{S}$ and Chambon R. Loss of uniqueness of numerical solutions of the borehole problem modelled with enhanced media. International Journal of Solids and Structures 2009; 46(17): 3173-3197.

24. Li J. A micromechanics-based strain gradient damage model for fracture prediction of brittle materials-part i: Homogenization methodology and constitutive relations. International Journal of Solids and Structures 2011; 48(24): 3336-3345.

25. Li J, Pham T, Abdelmoula $\mathrm{R}$ et al. A micromechanicsbased strain gradient damage model for fracture prediction of brittle materials - part ii: Damage modeling and numerical simulations. International Journal of Solids and Structures 2011; 48(24): 3346 - 3358.

26. Kotronis $P$, Chambon R, Mazars J et al. Local second gradient models and damage mechanics: application to concrete. In 11th international conference on fracture, Turin, Italy, Org. ICF, $c d$, paper, volume 5712. pp. 20-25.

27. Yang Y and Misra A. Micromechanics based second gradient continuum theory for shear band modeling in cohesive granular materials following damage elasticity. International Journal of Solids and Structures 2012; 49(18): 2500-2514.

28. Jouan G. Modélisation numérique de la localisation des déformations dans le béton avec un modèle de second gradient. Ph.d., Ecole Centrale de Nantes, Université de Liège (cotutelle), 2014. URL http://bictel.ulg.ac.be/ETD-db/collection/ available/ULgetd-11042014-112001/. (in French).

29. Jouan G, Kotronis P and Collin F. Using a second gradient model to simulate the behaviour of concrete structural elements. Finite Elements in Analysis and Design 2014; 90: 50 - 60. DOI: 10.1016/j.finel.2014.06.002.

30. Chambon R, Caillerie D and Matsuchima T. Plastic continuum with microstructure, local second gradient theories for geomaterials: localization studies. International Journal of Solids and Structures 2001; 38(46-47): 8503 - 8527. DOI: 10.1016/S0020-7683(01)00057-9.

31. Forest $\mathrm{S}$ and Sievert R. Elastoviscoplastic constitutive frameworks for generalized continua. Acta Mechanica 2003; 160(1-2): 71-111.

32. Zienkiewicz $\mathrm{O}$ and Taylor $\mathrm{R}$. The finite element method for solid and structural mechanics. Butterworth-Heinemann, 2005.

33. Zervos A, Papanastasiou $P$ and Vardoulakis I. A finite element displacement formulation for gradient elastoplasticity. International Journal for Numerical Methods in Engineering 2001; 50(6): 1369-1388. DOI:10.1002/1097-0207(20010228) 50:6<1369::AID-NME72>3.0.CO;2-K. 
34. Fischer P, Mergheim J and Steinmann P. On the $\mathrm{c} 1$ continuous discretization of non-linear gradient elasticity: A comparison of nem and fem based on bernstein-bézier patches. International Journal for Numerical Methods in Engineering 2010; 82(10): 1282-1307.

35. Shu J, King W and Fleck N. Finite elements for materials with strain gradient effects. International Journal for Numerical Methods in Engineering 1999; 44(3): 373-391.

36. Bésuelle P. Implémentation d'un nouveau type d'élément fini dans le code lagamine pour une classe de lois á longueur interne. Technical report, Laboratoire Sols, Solides Structures, Grenoble, France, 2003. Rapport d'activité.

37. Mindlin R. Second gradient of strain and surface-tension in linear elasticity. International Journal of Solids and Structures 1965; 1(4): 417-438.

38. Timoshenko S and Goodier JN. Theory of elasticity. New York McGraw-Hill - Engineering societies monographs, 1951.

39. Fernandes R, Chavant $C$ and Chambon R. A simplified second gradient model for dilatant materials: Theory and numerical implementation. International Journal of Solids and Structures 2008; 45(20): 5289 - 5307. DOI:10.1016/j.jjsolstr.2008.05.032.

40. Collin F, Caillerie D and Chambon R. Analytical solutions for the thick-walled cylinder problem modeled with an isotropic elastic second gradient constitutive equation. International Journal of Solids and Structures 2009; 46(22-23): 3927 - 3937. DOI:10.1016/j.ijsolstr.2009.05.017.

41. Pardoen B, Levasseur S and Collin F. Using local second gradient model and shear strain localisation to model the excavation damaged zone in unsaturated claystone. Rock Mechanics and Rock Engineering 2014; 48(2): 691-714.

42. Kotronis P. Stratégies de Modélisation de Structures en Béton Soumises à des Chargements Sévères. H.D.R. (Habilitation à Diriger des Recherches), Université Joseph-Fourier-Grenoble I, 2008. URL http://tel.archives-ouvertes.fr/ tel-00350461.

43. Ghavamian S, Carol I and Delaplace A. Discussions over meca project results. Revue Française de Génie Civil 2003; 7(5): $543-581$.

44. Jason L. Réponse au benchmark statique monotone du projet national ceos.fr, 2008. Rapport CEA SACLAY, DM2S/SEMT/LM2S, Rapport ANR MEFISTO.

45. Mazars J. Application de la mécanique de l'endommagement au comportement non linéaire et à la rupture du béton de structure. Thèse de doctorat d'état, Université Pierre et Marie Curie, Paris VI, 1984.

46. Cuvilliez S, Feyel F, Lorentz E et al. A finite element approach coupling a continuous gradient damage model and a cohesive zone model within the framework of quasi-brittle failure. Computer Methods in Applied Mechanics and Engineering
2012; 237: 244-259.

47. Simone A, Askes $\mathrm{H}$ and Sluys LJ. Incorrect initiation and propagation of failure in non-local and gradient-enhanced media. International Journal of Solids and Structures 2004; 41(2): 351-363.

48. Iacono C, Sluys LJ and van Mier JGM. Estimation of model parameters in nonlocal damage theories by inverse analysis techniques. Computer Methods in Applied Mechanics and Engineering 2006; 195(52): 7211-7222. DOI:10.1016/j.cma. 2004.12.033.

49. Iacono C, Sluys L and van Mier J. Calibration of a higher-order continuum model using global and local data. Engineering Fracture Mechanics 2008; 75(16): 4642-4665. DOI:10.1016/ j.engfracmech.2008.05.009.

50. Alam SY, Kotronis P and Loukili A. Crack propagation and size effect in concrete using a non-local damage model. Engineering Fracture Mechanics 2013; : 246-261DOI:10. 1016/j.engfracmech.2013.07.009.

51. Rolshoven S. Nonlocal plasticity models for localized failure. PhD Thesis, École Polytechnique fédérale de Lausanne, 2003. 